\title{
Unexpected Phenotype of STAT6 Heterozygous Mice Implies Distinct STAT6 Dosage Requirements for Different IL-4 Functions
}

\author{
Susanne Bürgis André Gessner \\ Institut für Klinische Mikrobiologie, Immunologie und Hygiene, Universität Erlangen-Nürnberg, \\ Erlangen, Germany
}

\section{Key Words}

IgE regulation • IL-4 signal transduction · STAT6

\begin{abstract}
Background: STAT6 is an important transcription factor in interleukin-4 (IL-4) signaling, a key cytokine in atopic diseases and allergic asthma. STAT6 gene-targeted mice are unable to develop IgE and Thelper 2 cell (Th2) responses in several models of allergic and infectious diseases. In experiments to further elucidate STAT6 functions in vivo, we unexpectedly observed severely impaired IL-4 functions in STAT6 heterozygous (STAT6+/-) mice which were further analyzed in this study. Methods: BALB/c mice, either wildtype (STAT6+/+), STAT6 heterozygous (STAT6+/-) or STAT6 deficient (STAT6-/-), were analyzed for their ability to mount an IL-4-induced IgE response in vitro and in vivo. Supernatants of stimulated B cells and sera of Leishmania major-infected mice were analyzed for $\lg \mathrm{E}, \lg \mathrm{G} 1$ and $\lg \mathrm{G} 2 \mathrm{a}$ concentrations by ELISA. Transcripts accompanying IgE class switching were amplified by RT-PCR and the expression of CD23 and MHC class II molecules on B cells was assessed by FACS analysis. Results: B cells from STAT6+/-mice were unable to secrete $\lg E$ in vitro and in vivo and transcripts accompanying IgE class switching were drastically reduced, whereas IL-4-induced upregulation of MHC class II was unimpaired and CD23 expression levels were only slightly affected. Additionally, STAT6+/- mice were equally
\end{abstract}

resistant to infection with L. major as STAT6-deficient (STAT6-/-) mice, due to a defect in mounting a Th2-dominated immune response. Conclusion: Different STAT6-dependent IL-4 functions require different thresholds of activated STAT6 molecules.

Copyright $\odot 2007$ S. Karger AG, Basel

\section{Introduction}

Interleukin-4 (IL-4) is a pleiotropic cytokine exerting various effects on different cell types [1]. Together with IL-13 it is the key cytokine in atopic diseases, e.g. allergic asthma. STAT6 is a transcription factor activated selectively by IL-4 and IL-13, which is the subject of ongoing research. The generation of STAT6 gene-targeted mice allowed to specifically analyze the role of this transcription factor [2-4]. Cells of STAT6 gene-targeted mice fail to upregulate IL-4-induced genes such as MHC II, CD23 or the IL- $4 \mathrm{R} \alpha$ chain upon IL-4 stimulation. Additionally, STAT6 KO mice cannot mount an efficient Thelper 2 cell (Th2) immune response and class switching of B cells towards IgE production is completely abrogated. As a result of these defects, mice show reduced peribronchial inflammation and airway hyperresponsiveness in models of allergic asthma $[5,6]$. The exact molecular mechanisms underlying these observations still remain incompletely understood.

\section{KARGER}

Fax +4161306 1234

E-Mail karger@karger.ch

www.karger.com (c) 2007 S. Karger AG, Basel

$1018-2438 / 07 / 1434-0263 \$ 23.50 / 0$

Accessible online at:

www.karger.com/iaa
Correspondence to: Prof. André Gessner

Institut für Klinische Mikrobiologie, Immunologie und Hygiene

Universität Erlangen-Nürnberg, Wasserturmstrasse 3, DE-91054 Erlangen (Germany)

Tel. +49 9131852 2580, Fax +499131 8525991

E-Mail gessner@mikrobio.med.uni-erlangen.de 
To further address the role of STAT6 in IL-4-dominated immune responses, we included STAT $6+/-$ mice as a control group in experiments with STAT6 ${ }^{\text {tm1Gru }}$ genetargeted miceand wild-typemice.Surprisingly, STAT6+/mice displayed a phenotype similar to STAT6-/- mice for some (IgE expression, antiparasitic immune response), but not all (enhanced expression of CD23 and MHC class II molecules on IL-4-stimulated B cells) IL-4-mediated functions analyzed. This finding argues for hitherto unknown distinct thresholds of activated STAT6 molecules for different IL-4-mediated functions.

\section{Material and Methods}

Animals

$\mathrm{BALB} / \mathrm{c}$ mice and mice heterozygous or deficient for STAT6 $^{\text {tmlGru }}$ [2] were bred in our animal facility under specific pathogen-free conditions. Female mice between 6-8 weeks of age were used for the experiments according to the guidelines for animal welfare and legal requirements.

\section{In vitro Class Switching}

Single cell suspensions from spleens were enriched for B cells by depletion of cells positive for CD3, CD11b, Gr-1 and NK DX-5 using the magnetic labeling and separation protocol (BD Biosciences, Heidelberg, Germany) according to the manufacturer's recommendation. For class switching of splenic B cells towards IgE production, $1 \times 10^{6}$ cells per well ( 24 -well plate, culture volume $1 \mathrm{ml}$ ) were cultured either with medium alone or in the presence of $\alpha$-CD40 (FGK) antibody with or without $20 \mathrm{ng} / \mathrm{ml}$ recombinant IL-4 (Strathmann, Hamburg, Germany). Supernatants were collected on days 3, 5, 7 and 9 for IgE ELISA. On day 2 and 4 total RNA was isolated (RNeasy mini kit, Qiagen) from stimulated cells $(\alpha-\mathrm{CD} 40+\mathrm{IL}-4)$, and after reverse transcription with oligo-dT primers (SuperScript II RNase, Invitrogen) samples were analyzed for the presence of switch transcripts (germ line transcript: IeF and CeR, $392 \mathrm{bp}$; postswitch transcript: ImF and CeR, 285 bp; IeF: 5'-TGGGATCAGATCTTTGAG-3', CeR: 5'-CCAGGGTCATGGAAGCAGTG-3', ImF: 5'-CTCTGGCCCTGCTTATTGTTG-3'; PCR conditions: 37 cycles, annealing: $45 \mathrm{~s}, 65^{\circ} \mathrm{C}$, elongation: $45 \mathrm{~s}, 72^{\circ} \mathrm{C}$ ). Cells were analyzed by FACS analysis on day 4 for surface molecules CD23 and MHC II with antibodies from Pharmingen (BD Biosciences).

\section{In vivo Class Switching}

Mice on a susceptible BALB/c background were infected subcutaneously with $2 \times 10^{6}$ promastigotes (Leishmania major strain MHOM/IL/81/FEBNI) in the right hind footpad. Footpad swelling was monitored over time. On day 32 mice were sacrificed and sera were collected for ELISAs.

\section{ELISAs}

Sandwich ELISAs for total IgE and Leishmania-specific IgG1 and IgG2a were performed using the following antibodies: capture IgE: clone R35-72, $2 \mu \mathrm{g} / \mathrm{ml}$ (Pharmingen); biotinylated IgE for detection: clone R35-118, $2 \mu \mathrm{g} / \mathrm{ml}$ (Pharmingen); capture
Leishmania-specific antibodies: preparation of total antigen $(1 \times$ $10^{9}$ Leishmania/ml, repeated freeze-thaw cycles, dilution 1:200); biotinylated IgG1 for detection: clone G1-6.4, diluted 1:1,000 (Pharmingen), and biotinylated IgG2a for detection: clone R1915, diluted 1:1,000 (Pharmingen). Biotinylated detection antibodies were coupled to HRP with the StrepAB-Complex (Dako Cytomation), and TMB (Sigma) was added as substrate.

\section{Western Blot}

Whole cell lysates of spleens from BALB/c wild-type, STAT6 heterozygous and STAT6-deficient mice were prepared in RIPA lysis buffer ( $150 \mathrm{~mm} \mathrm{NaCl}, 1.0 \%$ Igepal CA-630, 0.5\% sodium deoxycholate, $0.1 \%$ SDS, $50 \mathrm{~mm}$ Tris, $\mathrm{pH} 8.0$ ). $40 \mu \mathrm{g}$ of total protein per sample were applied to a polyacrylamide gel, and after blotting proteins were detected with specific antibodies: M-20 (1 $\mu \mathrm{g} /$ $\mathrm{ml}$, Santa Cruz), M-200 (1 $\mu \mathrm{g} / \mathrm{ml}$, Santa Cruz) or anti-actin A2066 (1:250; Sigma). The secondary antibody (donkey-anti-rabbit-POX from Dianova) was diluted 1:10,000 and blots were developed with the ECL Plus Western Blotting Detection System from Amersham. Quantification was performed with the AIDA software v4.03.031 (Raytest Isotopenmessgeräte, Straubenhardt, Germany).

Statistics

Data were analyzed with either two-tailed t test or one-way ANOVA using GraphPad Prism version 3.00 for Windows (GraphPad Software, San Diego, Calif., USA; www.graphpad.com).

\section{Results and Discussion}

In the present study we investigated IL-4-induced effects in STAT6+/- mice (Stat6 ${ }^{\text {tmlGru}}$ ) in comparison to wild-type and STAT6-deficient animals. Splenic B cells were isolated and stimulated in vitro with $\alpha$-CD40 + IL-4. Upregulation of the activation markers MHC II and the low affinity IgE receptor CD23 on stimulated B cells was assessed by FACS analysis. Mean fluorescence intensities $( \pm S D$ of at least three independent experiments) for MHC II expression were $547 \pm 129$ compared to $1,646 \pm 189$ for STAT6+/+ samples, $619 \pm$ 93 compared to $1,776 \pm 87$ for STAT6+/- samples and $558 \pm 48$ compared to $548 \pm 56$ for STAT6-/- samples, comparing $\alpha$-CD40 stimulation with $\alpha$-CD40 + IL-4 stimulation. Mean fluorescence intensities for CD23 expression were $88 \pm 7$ compared to $161 \pm 8$ for STAT $6+/+$ samples, $77 \pm 16$ compared to $116 \pm 20$ for STAT $6+/-$ samples and $77 \pm 16$ compared to $85 \pm 8$ for STAT6-/- samples, comparing $\alpha$-CD40 stimulation with $\alpha-C D 40+$ IL- 4 stimulation. So cells from STAT $6+/-$ mice did upregulate MHCII expression to a similar extent as and CD23 expression to only a slightly reduced extent compared to cells from STAT $6+/+$ mice, whereas cells from STAT6-/- mice showed no upregulation of 


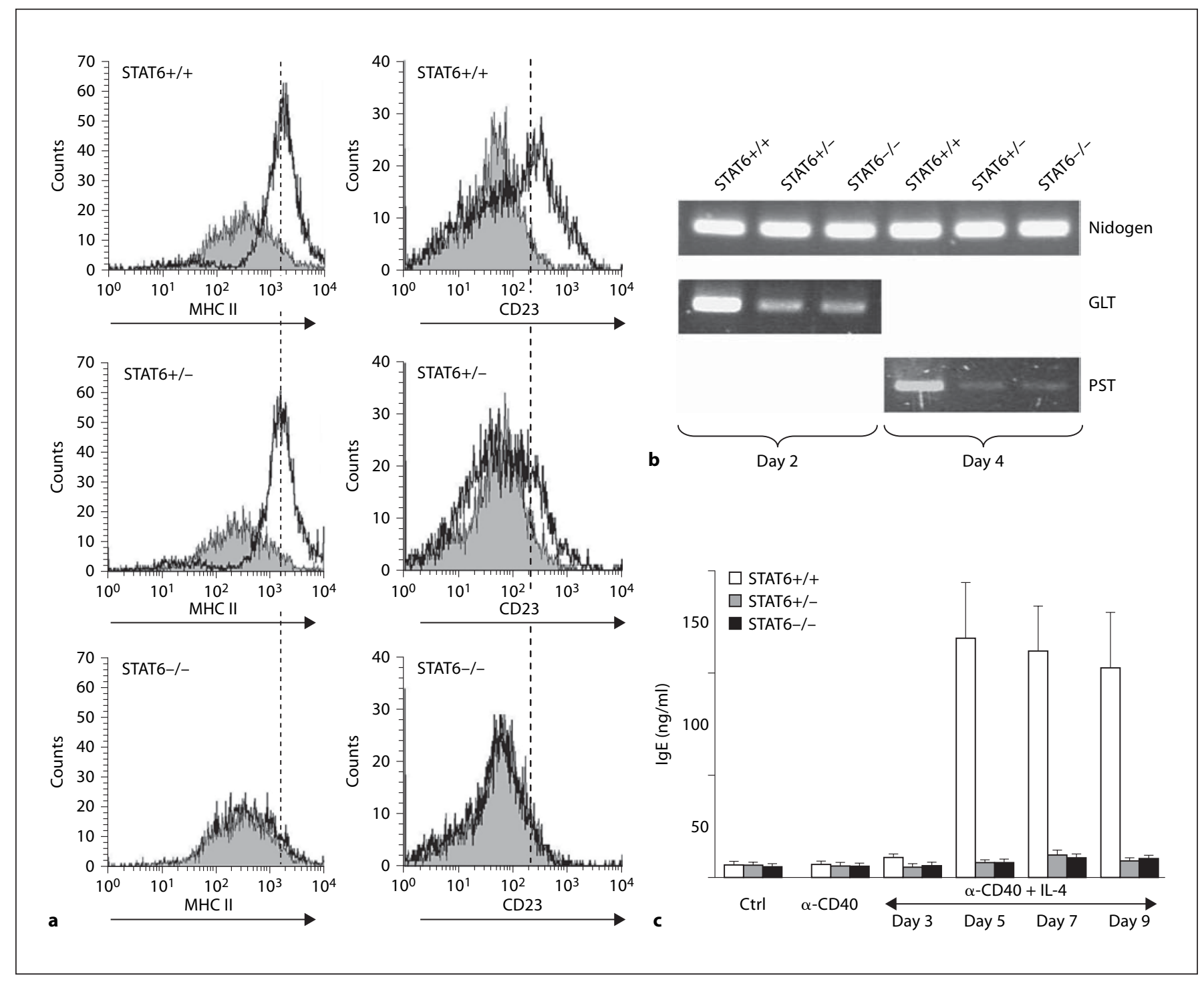

Fig. 1. IgE class switching of splenic B cells in vitro. Splenocytes from BALB/c mice either wild type $(+/+)$, STAT6 heterozygous $(+/-)$ or STAT6 deficient (-/-) were enriched for B cells by negative depletion of non-B cells. a Cells were stimulated with $\alpha$-CD40 (closed lines) or $\alpha$-CD40 + IL-4 (open lines). On day 4 cells were analyzed for the upregulation of activation markers CD23 and MHC II by FACS. Data are representative of three independent experiments. b Cells were stimulated with $\alpha$-CD $40+$ IL- 4 . Total RNA was isolated on days 2 and 4 . After reverse transcription,
cDNA was subjected to PCR analysis to amplify germ line transcripts (GLT) or postswitch transcripts (PST) after 2 or 4 days of stimulation, respectively. Data are representative of three independent experiments. c Cells were stimulated with $\alpha$-CD $40, \alpha-$ CD40 + IL- 4 or were left unstimulated (Ctrl). Supernatants were collected on days 3, 5, 7 and 9, and were analyzed by ELISA for IgE concentrations. Data are expressed as mean $\pm \operatorname{SEM}(n=6) . p<$ 0.001 on days 5,7 and 9 . these molecules, as already described for the latter molecule [2] (fig. 1a).

Class switching of stimulated B cells towards IgE production is preceded by the generation of a sterile germline transcript originating from the I $\varepsilon$ promoter [7]. After class switch recombination, a postswitch transcript di- rected by the I $\mu$ promoter is detectable [8]. Both the germ line transcript generated from the original configuration of the Ig heavy chain locus as well as the postswitch transcript generated from the Ig heavy chain locus after class switch recombination were detectable in cDNA samples from IL-4-stimulated BALB/c STAT6+/+ B cells after 2 


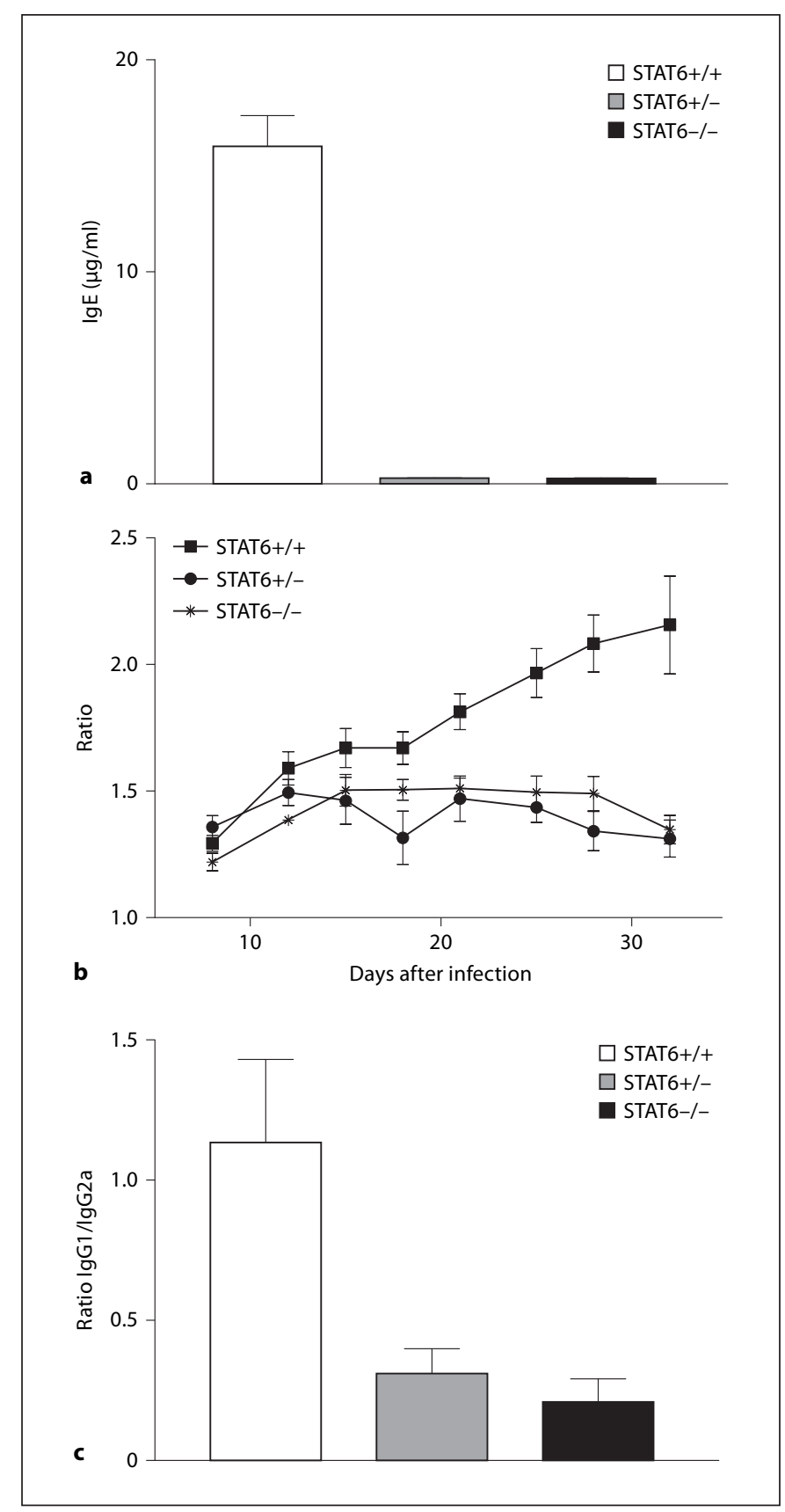

Fig. 2. Mice on a susceptible BALB/c background, either wild type $(+/+)$, STAT6 heterozygous $(+/-)$ or STAT6 deficient $(-/-)$, were infected with $2 \times 10^{6}$ L. major promastigotes by subcutaneous injection into the right hind footpad. a Mice were bled on day 32 after infection and sera were analyzed by ELISA for IgE. $\mathrm{p}<0.001$ wild type vs. heterozygous. b Footpad swelling was monitored over time. The thickness of the infected footpad vs. the uninfected footpad (ratio) is a measure for the severity of infection and inflammation. c Sera collected from infected mice on day 32 after infection were analyzed by ELISA for Leishmania-specific IgG1 and IgG2a antibodies. The ratio of IgG1 vs. IgG2a titers is depicted, being indicative of a Th2-dominated immune response. $\mathrm{p}<0.006$ wild type vs. heterozygous. Data are expressed as mean $\pm \operatorname{SEM}(n=6)$. or 4 days, respectively. In contrast, these transcripts were strongly reduced in samples from STAT6+/- B cells. Most probably this residual transcriptional activity is due to $\alpha$ CD40 stimulation, as it does not exceed the level of transcription observed in STAT6-deficient mice (fig. 1b) [9]. Consistent with this observation, barely any IgE was detectable in supernatants of $\alpha$-CD $40+$ IL-4-stimulated B cells in STAT6+/- and STAT6-/- samples (fig. 1c), reflecting the inability of STAT6+/- B cells to undergo IgE class switching upon IL-4 stimulation.

To confirm these in vitro data in an in vivo setting, we infected mice on a susceptible BALB/c background subcutaneously with the intracellular parasite L. major, an infection known to result in a Th2 cell-dominated immune response with high serum levels of $\operatorname{IgE}[10,11]$. Sera collected on day 32 postinfection exhibited high levels of IgE in STAT $6+/+$ mice $(>15 \mu \mathrm{g} / \mathrm{ml})$, whereas equally low levels $(<300 \mathrm{ng} / \mathrm{ml})$ were detected in sera of STAT6+/- and STAT6-/- mice (fig. 2a). This finding confirmed the inability of STAT $6+/-$ mice to mount a pronounced IgE response in an in vivo setting. Additionally, STAT6+/-BALB/c mice were able to control infection with L. major (fig. 2b), reflecting their inability to mount an IL-4-driven Th2-dominated immune response, which is responsible for the fatal outcome of this infection. To further substantiate this observation, we determined titers of Leishmania-specific IgG1 and IgG2a antibodies, being characteristic for a Th2- or a Th1-dominated immune response, respectively. A high ratio of IgG1 versus IgG2a titers is indicative of a Th2-biased immune response. STAT6+/+ mice exhibited a significantly higher IgG1/IgG2a ratio than both STAT6+/- and STAT6-/- mice, again arguing for an inefficient generation of a Th2-dominated immune response in STAT6+/mice (fig. 2c).

STAT $6^{\text {tm } 1 \text { Gru }}$ KO mice were generated by replacing exons encoding amino acids 505-584 with a neomycin resistance cassette, thereby deleting the genomic region coding for parts of the $\mathrm{SH} 2$ domain required for dimerization and functionality [2]. As we observed such marked phenotypes in heterozygous mice, we investigated whether a mutant STAT6 protein might be expressed from the targeted locus, possibly acting as a dominant negative protein and thereby abrogating STAT6 functionality in the heterozygous configuration. To further investigate the presence of such a truncated STAT6 protein, we analyzed cell lysates from mice either STAT $6+/+$, STAT6+/- or STAT6-/- in Western blot experiments using two different STAT6 antibodies directed against different domains of the protein. An antibody directed 
Fig. 3. Western blot analysis of whole cell lysates of wild-type $(+/+)$, STAT6 heterozygous (+/-) and STAT6-deficient (-/-) mice. Proteins were extracted from single cell suspensions of splenocytes with RIPA lysis buffer. $40 \mu \mathrm{g}$ of total protein were separated on a polyacrylamide gel, and after Western blotting, blots were probed with STAT6-specific antibodies either recognizing the C-terminus of STAT6 (M20) (a) or amino acids $280-480$ of the STAT6 protein (M200) (b). Blots were reprobed with an actin-specific antibody as a loading control.

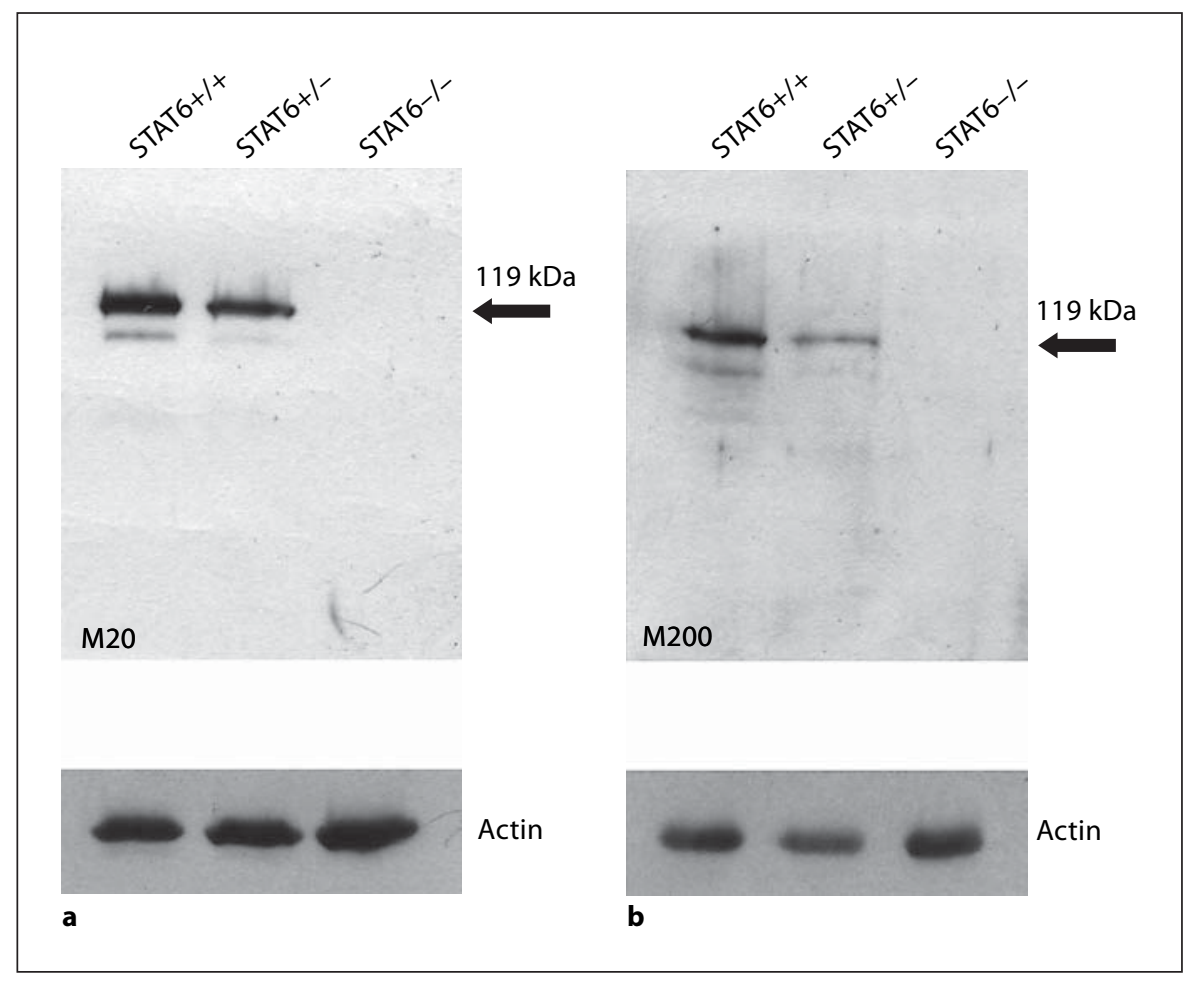

against the C terminus of STAT6 (M20) detected the protein in STAT6+/+ and STAT6+/- samples, whereas in STAT6 KO mice no protein was detectable (fig. 3a). The relative amounts of STAT6 protein (normalized to actin) were estimated to be $56.0 \%$ and not detectable $(<1.5 \%)$ of the protein amount detectable in the STAT $6+/+$ configuration for STAT6+/- and STAT6-/- samples, respectively. This observation indicates that reduced STAT6 levels might be responsible for the phenotype observed in STAT6+/- mice. The second antibody raised against amino acids 280-480 (M200), a region upstream of the STAT6 gene disruption, also detected the wild-type protein of approximately $119 \mathrm{kDa}$, but failed to detect any truncated protein with a theoretical size of $54 \mathrm{kDa}$ in STAT6+/- or STAT6-/- samples (fig. 3b). As no evidence for a truncated version of STAT6 was obtained from these experiments, we consider it unlikely that such a molecule might be responsible for the observed phenotype of STAT6+/- mice. However, in humans, a naturally occurring STAT6 splice variant has been described that lacks 33 amino acids in the SH2 domain of STAT6 and that functions as a dominant negative species [12]. The gene-targeted STAT6 ${ }^{\text {tmlGru }}$ locus lacks exons encoding amino acids 505-584 of STAT6, which corresponds to 80 amino acids including those from the human dom- inant negative splice variant. Therefore, a truncated protein being present in the heterozygous configuration might theoretically be responsible for the observed deficiencies in STAT6+/- mice, but should have been detected by the STAT6-specific M200 antibody. Irrespective of these considerations, different IL-4-induced STAT6-dependent functions obviously display distinct STAT6 dosage requirements. Whether this is due to either a complex situation involving so far undetectable dominant negative STAT6 molecules, or - more likely - to a simple reduction of STAT6 concentrations in the heterozygous configuration is subject of ongoing experiments in our laboratory.

\section{Acknowledgments}

We thank Rimma Husch for excellent technical assistance and Gerhard Groer for helpful discussions. This work was supported by the DFG (SFB 643, B1 and GRK 592). 


\section{References}

1 Nelms K, Keegan AD, ZamoranoJ, Ryan JJ, Paul WE: The IL-4 receptor: signaling mechanisms and biologic functions. Annu Rev Immunol 1999;17:701-738.

2 Kaplan MH, Schindler U, Smiley ST, Grusby MJ: Stat 6 is required for mediating responses to IL- 4 and for development of Th2 cells. Immunity 1996;4:313-319.

3 Takeda K, Tanaka T, Shi W, Matsumoto M, Minami M, Kashiwamura S, Nakanishi K, Yoshida N, Kishimoto T, Akira S: Essential role of Stat6 in IL-4 signalling. Nature 1996; 380:627-630

4 Shimoda K, van Deursen J, Sangster MY, Sarawar SR, Carlson RT, Tripp RA, Chu C, Quelle FW, Nosaka T, Vignali DA, Doherty PC, Grosveld G, Paul WE, Ihle JN: Lack of IL-4-induced Th2 response and IgE class switching in mice with disrupted Stat6 gene. Nature 1996;380:630-633.
5 Akimoto T, Numata F, Tamura M, Takata Y, Higashida N, Takashi T, Takeda K, Akira S: Abrogation of bronchial eosinophilic inflammation and airway hyperreactivity in signal transducers and activators of transcription (STAT)6-deficient mice. J Exp Med 1998;187:1537-1542.

6 Kuperman D, Schofield B, Wills-Karp M, Grusby MJ: Signal transducer and activator of transcription factor 6 (Stat6)-deficient mice are protected from antigen-induced airway hyperresponsiveness and mucus production. J Exp Med 1998;187:939-948.

7 Stavnezer-Nordgren J, Sirlin S: Specificity of immunoglobulin heavy chain switch correlates with activity of germline heavy chain genes prior to switching. EMBO J 1986;5:95102.

8 Li SC, Rothman PB, Zhang J, Chan C, Hirsh D, Alt FW: Expression of I mu-C gamma hybrid germline transcripts subsequent to immunoglobulin heavy chain class switching. Int Immunol 1994;6:491-497.
9 Linehan LA, Warren WD, Thompson PA, Grusby MJ, Berton MT: STAT6 is required for IL-4-induced germline Ig gene transcription and switch recombination. J Immunol 1998;161:302-310.

10 Sadick MD, Heinzel FP, Holaday BJ, Pu RT, Dawkins RS, Locksley RM: Cure of murine leishmaniasis with anti-interleukin 4 monoclonal antibody. Evidence for a T cell-dependent, interferon gamma-independent mechanism. J Exp Med 1990;171:115-127.

11 Sacks D, Noben-Trauth N: The immunology of susceptibility and resistance to Leishmania major in mice. Nat Rev Immunol 2002;2: 845-858.

12 Patel BK, Pierce JH, LaRochelle WJ: Regulation of interleukin 4-mediated signaling by naturally occurring dominant negative and attenuated forms of human Stat6. Proc Natl Acad Sci USA 1998;95:172-177. 